Check for updates

Cite this: Analyst, 2020, 145, 1825

\section{A twin enrichment method based on dispersive liquid-liquid microextraction and field-amplified sample injection for the simultaneous determination of sulfonamides $\uparrow$}

\author{
Suya Ma, ${ }^{a, b}$ Shixuan Yang, ${ }^{a}$ Zhihua Song, ${ }^{c}$ Jinhua Li, ${ }^{\star a}$ Qiaocui Shi, ${ }^{d}$ Huiyan You, ${ }^{* b}$ \\ Huitao Liu, ${ }^{e}$ Min Lva and Lingxin Chen (iD *a
}

\begin{abstract}
A twin enrichment method based on offline dispersive liquid-liquid microextraction (DLLME) coupled with online field-amplified sample injection (FASI) was developed for the simultaneous determination of four sulfonamide (SA) antibiotics, including sulfamethazine (SMZ), sulfamerazine (SMR), sulfadizine (SDZ) and sulfacetamide (SFA), in different environmental waters, followed by capillary electrophoresis (CE). Various parameters that affected the separation performance of CE and the enrichment efficiencies of DLLME and FASI were optimized in detail, and excellent CE separation was attained within 6 min. The DLLME-FASI-CE offered high sensitivity enrichment factors of 206, 166, 185 and 150 for SMZ, SMR, SDZ and SFA, respectively. Highly sensitive detection was realized with low limits of detection (LODs), which ranged from 2.0-23.0, 2.2-26.0 and 4.3-63.0 $\mathrm{ng} \mathrm{mL}^{-1}$ in tap water, lake water and seawater, respectively, as well as limits of quantification (LOQs) within 6.0-63.0, 7.4-96.0 and 14.0-201.0 ng mL $\mathrm{mL}^{-1}$, respectively. Satisfactory recoveries in the range of $91-108 \%$ were obtained with the three spiked environmental water samples, and the relative standard deviations were from 1.09-7.45\%. The simple effective twin enrichment method provided promising perspective for CE determination of SAs in complicated aqueous matrices, with rapidity, sensitivity, and accuracy.
\end{abstract}

Received 24th October 2019, Accepted 11th January 2020

DOI: $10.1039 / c 9 a n 02127 b$

rsc.li/analyst animal original should not exceed $100 \mu \mathrm{g} \mathrm{kg}^{-1}$, as stipulated by the European Union (EU) and China. ${ }^{4}$ Due to the slow degradation and long residual time of SAs in the environment, their metabolites often exist in environmental waters for a long time, especially sulfamethazine (SMZ), sulfamerazine (SMR), sulfadizine (SDZ) and sulfacetamide (SFA). ${ }^{5}$ If humans ingest SA-contaminated waters or animal tissues, it may increase the risk of allergies, carcinogenesis, and the formation of resistant bacteria in the human body. ${ }^{6}$ Thus, it is imperative to develop sensitive methodologies for the separation and determination of SA residues in complicated environmental waters.

In particular, SA residues in environmental waters are commonly present at (ultra)trace levels which are lower than the limit of detection of the most analytical instruments and thereby influential sample preparation is required for SA analysis. In this regards, a variety of sample preparation methods such as salting-out liquid-liquid extraction (SLLE), ${ }^{7}$ polymer monolith microexcation, ${ }^{8}$ solid-phase extraction (SPE), ${ }^{9-11}$ and dispersive liquid-liquid microextraction (DLLME) ${ }^{12}$ have been reported for the pre-concentration of SAs. Among them, DLLME is an ideal technique because of its simplicity, low 
cost, effectiveness, speed, and the small consumption of organic solvents. ${ }^{12-19}$ Moreover, compared with SPE based approaches which require tedious adsorbents preparation steps, DLLME can run quickly in general laboratory by only routine glassware and a few microliters of organic solvents. It is well known that the figures of merits of analytical approaches are extremely dependent on their corresponding enrichment efficiencies. In other words, while the concentration of the analyte(s) in the elution is increased compared with the sample solution, the enrichment factor is enhanced and lower limits of detection (LODs) can be achieved. Therefore, it is highly desirable to enhance this important parameter. Respect to this aim, twin/multiple sample preparations conjoining and hyphenated sample treatments have been utilized. ${ }^{20,21}$ However, arranging two or more enrichment methods is not always easy and effective, since in each adsorption/desorption cycle some of the analyte molecules can be lost and lead to the decrease of recovery.

Undoubtedly, online enrichment procedure is more preferable compared with offline mode owing to some remarkable superiority, such as the entire process is performed under controlled conditions, minimizing human and systematic errors. Capillary electrophoresis (CE) has unique online enrichment modes, viz., online stacking, which can be easily realized by simply adjusting the background electrolyte (BGE) composition and the sample injection procedure. ${ }^{22,23}$ Generally, the CE online stacking includes four basic modes, that is, field amplication (FA), sweeping, $\mathrm{pH}$ regulation, and isotachophoresis (ITP). ${ }^{22}$ Specifically, field-amplified sample injection (FASI), ${ }^{23,24}$ sweeping, ${ }^{25,26}$ dynamic $\mathrm{pH}$ junction ${ }^{27,28}$ and transient isotachophoresis (tITP) ${ }^{29,30}$ are widely utilized for CE determination of various analytes. Amongst them, FASI combines electrophoresis and electroosmotic flow (EOF), and allows ions to enter the capillary under the action of an electric field to achieve stacking. ${ }^{22-24}$ FASI enables the concentration of analytes at the capillary inlet, which is not limited by the capillary length in stacking technology. Pressure is added to the injection to counter the flow caused by the EOF. ${ }^{23}$ The method can be readily carried out and provide high enhancement in concentration, without adverse impacts on peak shape and resolution. ${ }^{23,31}$ On the other hand, besides other chromatography techniques such as liquid chromatography-mass spectrometry (LC-MS), ${ }^{32,33}$ high-performance liquid chromatography (HPLC), ${ }^{34,35}$ and thin layer chromatography, ${ }^{36} \mathrm{CE}$ has also often been used for SA residue analysis, due to its high separation efficiency, short analysis time and small volume requirements of samples and reagents. ${ }^{12,37,38}$

Up to know, various practical methods have been reported for the SA determination in different matrices. For example, Xia et al. have synthesized a magnetic, mesoporous core/shell structured $\mathrm{Fe}_{3} \mathrm{O}_{4}$ @JUC-48 nanocomposite and employed it as a magnetic solid phase extraction (MSPE) sorbent for the determination of trace SAs in meat samples. ${ }^{1}$ Yu et al. have developed in-syringe ionic liquid assisted DLLME and applied it for the extraction of SAs in blood followed by HPLC determination. ${ }^{9}$ Ben et al. have used SPE-LC-MS for simultaneous determination of three classes of commonly used veterinary antibiotics including five SAs, three tetracyclines and one macrolide in swine wastewaters. ${ }^{11}$ However, the introduction of sensitive, selective, inexpensive, straightforward, and quick approaches still is in high demand.

Therefore, in this study, we proposed an offline-online twin enrichment method by combining DLLME and FASI for the simultaneous enrichment of SAs in environmental waters followed by determination using $\mathrm{CE}$, including SMZ, SMR, SDZ and SFA. The main parameters influencing DLLME-FASI-CE performance were investigated in a univariate fashion, such as the properties of running buffer, organic modifier and applied voltage for $\mathrm{CE}$ separation, injection voltage, injection time and water plug for FASI, as well as the properties of extraction/disperser solvents and extraction time for DLLME. Under optimal conditions, the DLLME-FASI-CE method was well validated and successfully applied for the determination of SAs in different environmental water samples.

\section{Experimental}

\subsection{Reagents and samples}

HPLC grade SA standards, including sulfamethazine (SMZ), sulfamerazine (SMR), sulfadizine (SDZ) and sulfacetamide (SFA), were provided from Sigma-Aldrich (Steinheim, Germany) and their structures were shown in Fig. S1. $\dagger$ Standard solutions containing $1.0 \mathrm{mg} \mathrm{mL}^{-1}$ of each SA were prepared by dissolving the required amounts of the standard in methanol $(\mathrm{MeOH})$. They were stored in a refrigerator at $4{ }^{\circ} \mathrm{C}$ until use. All chemicals such as sodium dihydrogen phosphate $\left(\mathrm{NaH}_{2} \mathrm{PO}_{4}\right)$, phosphoric acid $\left(\mathrm{H}_{3} \mathrm{PO}_{4}\right)$, sodium chloride $(\mathrm{NaCl})$ and sodium hydroxide $(\mathrm{NaOH})$ were of analytical grade and were obtained from Sinopharm Chemical Reagent (Shanghai, China). Chromatographic grade acetonitrile (ACN), $\mathrm{MeOH}$, dimethyl sulfoxide (DMSO) and chlorobenzene $\left(\mathrm{C}_{6} \mathrm{H}_{5} \mathrm{Cl}\right)$ were purchased from J\&K Chemical (Beijing, China). The other analytical grade chemicals, such as dichloromethane $\left(\mathrm{CH}_{2} \mathrm{Cl}_{2}\right)$ and chloroform $\left(\mathrm{CHCl}_{3}\right)$, were purchased from Sinopharm Chemical Reagent (Shanghai, China). Carbon tetrachloride $\left(\mathrm{CCl}_{4}\right)$ was obtained from Aladdin (Shanghai, China). Ultrapure water with the specific resistance of $18.2 \mathrm{M} \Omega \mathrm{cm}$ was produced by Pall Cascada ${ }^{\mathrm{TM}}$ lab water purification system (Pall Corp., Massachusetts, USA) for aqueous solution preparation throughout the study.

The lake water sample was collected from an artificial lake located in the schoolyard of Yantai University, and the seawater sample was collected near freshwater fisheries in the Huanghai Sea. Lake water and seawater were stored in the dark at $4{ }^{\circ} \mathrm{C}$ until use. The tap water sample was obtained in the laboratory as needed. Before use, all the water samples were passed through microporous nylon filters with pore sizes of $0.45 \mu \mathrm{m}$ in diameter. Several aliquots from $5 \mathrm{~mL}$ filtered water samples were spiked with the SA standards with different concentrations and this was followed by the DLLME-FASI-CE procedure. 


\subsection{Instrumentation}

All electrophoresis experiments were performed on a SCIEX P/ ACETM MDQ plus CE system (Fullerton, CA, USA) equipped with a DAD. Data were collected using a Beckman P/ACE workstation version 32 Karat software. The $\mathrm{pH}$ of the buffer solution was calibrated by a pHS-3C digital pH meter (Hangzhou Dongxing Instrument Factory, Hangzhou, China).

\subsection{CE conditions}

Bare fused-silica capillaries, with an i.d. of $75 \mu \mathrm{m}$, o.d. of $375 \mu \mathrm{m}$, total length of $50.2 \mathrm{~cm}$ and effective length of $40 \mathrm{~cm}$ (Yongnian Photoconductive Fiber Factory, Hebei, China), were used throughout the entire experiment. A new capillary was initialized by rinsing with water for $10 \mathrm{~min}, 1.0 \mathrm{M} \mathrm{NaOH}$ for $40 \mathrm{~min}$, water for $10 \mathrm{~min}$ and running buffer for $30 \mathrm{~min}$ before use. The capillary was rinsed with running buffer for $3 \mathrm{~min}$ between analyses. All solutions were filtered through microporous nylon filters with pore diameters of $0.45 \mu \mathrm{m}$ before use. The detection wavelength was set at $254 \mathrm{~nm}$. The injection pressure was applied at 0.5 psi for $5 \mathrm{~s}$, known as hydrodynamic injection (HDI), and the separation voltage was set at $20 \mathrm{kV}$. The running buffer consisted of $20 \mathrm{mmol} \mathrm{L}{ }^{-1} \mathrm{NaH}_{2} \mathrm{PO}_{4}$ and $10 \% \mathrm{ACN}(\mathrm{v} / \mathrm{v})$, adjusted to $\mathrm{pH} 7.8$ with $1 \mathrm{M} \mathrm{NaOH}$.

\subsection{DLLME-FASI-CE procedure}

First, the DLLME procedure was carried out. $5 \mathrm{~mL}$ of aqueous solution containing SMZ, SMR, SDZ and SFA was placed into a $10 \mathrm{~mL}$ conical tube centrifuge. A mixture of $\mathrm{C}_{6} \mathrm{H}_{5} \mathrm{Cl}$ (extraction solvent, $400 \mu \mathrm{L}$ ) and DMSO (disperser solvent, $800 \mu \mathrm{L}$ ) was rapidly injected into the aqueous sample using a syringe and then the mixture was gently shaken by hand. $\mathrm{C}_{6} \mathrm{H}_{5} \mathrm{Cl}$ was dispersed throughout the aqueous phase and a cloudy solution of fine droplets was formed. After the cloudy solution was centrifuged for $20 \mathrm{~min}$ at $2500 \mathrm{rpm}$, the extracting solvent containing the analytes was sedimented at the bottom of the tube. The organic phase is dried in a vacuum drying oven for later use. The final residues were redissolved using $50 \mu \mathrm{L} \mathrm{MeOH} /$ $\mathrm{H}_{2} \mathrm{O}(\mathrm{v} / \mathrm{v}=1: 1)$ for injection into the capillary. Then, FASI was performed as follows: $3 \mathrm{~s}$ of injection time of water plug for $0.507 \mathrm{~cm}, 30 \mathrm{~s}$ of electrokinetic injection $(-10 \mathrm{kV})$ of the sample and an assisted pressure of $0.5 \mathrm{psi}$, followed by $\mathrm{CE}$ determination. Fig. 1 schematically shows the DLLME-FASI-CE procedure.

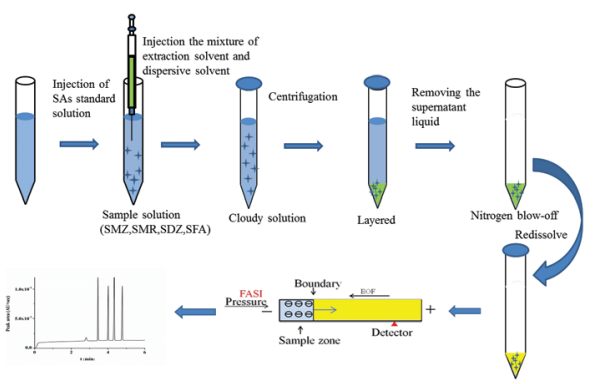

Fig. 1 Schematic of the DLLME-FASI-CE procedure.

\subsection{Sensitivity enrichment factor (SEF)}

Sensitivity enrichment factor (SEF) was calculated as follows,

$$
\mathrm{SEF}=C_{1} / C_{0}
$$

where $C_{0}$ and $C_{1}$ are the initial concentration of analytes in the aqueous solution and the concentration of analytes after enrichment (by DLLME, FASI or DLLME-FASI), respectively.

\section{Results and discussion}

\subsection{Optimization of $\mathrm{CE}$ conditions}

In this work, capillary zone electrophoresis (CZE), the most simple and commonly utilized CE mode, was adopted for the separation of the four SAs, based on our research experiences $^{12,39}$ and by referring to other groups' study. ${ }^{40,41}$ The factors that influenced CZE separation were optimized, including type, concentration and $\mathrm{pH}$ of the buffer solution, type and content of the organic modifier, the content of $\mathrm{NaCl}$ and the applied voltage.

The selection of BGE has an important influence on migration behavior. Two types of commonly used separation buffer solutions were investigated, including $\mathrm{Na}_{2} \mathrm{~B}_{4} \mathrm{O}_{7} \cdot 10 \mathrm{H}_{2} \mathrm{O}$ and $\mathrm{NaH}_{2} \mathrm{PO}_{4}$. The results showed that the $\mathrm{Na}_{2} \mathrm{~B}_{4} \mathrm{O}_{7} \cdot 10 \mathrm{H}_{2} \mathrm{O}$ buffer could not effectively separate, while the $\mathrm{NaH}_{2} \mathrm{PO}_{4}$ buffer could separate the four SAs, possibly because $\mathrm{Na}_{2} \mathrm{~B}_{4} \mathrm{O}_{7} \cdot 10 \mathrm{H}_{2} \mathrm{O}$ led to a slight broadening of the peaks. Next, different concentrations of $\mathrm{NaH}_{2} \mathrm{PO}_{4}$ buffer $\left(10,20,30,40 \mathrm{mmol} \mathrm{L}{ }^{-1}\right)$ were optimized. As shown in Fig. $\mathrm{S} 2, \uparrow$ high buffer concentrations caused the baseline to be unstable. The possible reason is that a high buffer concentration can produce a high zeta potential and thereby a high EOF, easily resulting in incomplete baseline separation. ${ }^{13}$ Therefore, $20 \mathrm{mmol} \mathrm{L}{ }^{-1} \mathrm{NaH}_{2} \mathrm{PO}_{4}$ was selected.

To obtain high separation efficiency within a relatively short time, the $\mathrm{pH}$ of the running buffer should be optimized. In this study, $20 \mathrm{mmol} \mathrm{L}{ }^{-1} \mathrm{NaH}_{2} \mathrm{PO}_{4}$ solutions with different $\mathrm{pH}$ values $(3.2,4.8,7.8$, and 11.8$)$ were investigated. As shown in Fig. $\mathrm{S} 3, \dagger$ when the $\mathrm{pH}$ value was 3.2, no peaks of the four SAs were found. With an increase in $\mathrm{pH}$ value, the separation degree of the SAs increased. However, when the $\mathrm{pH}$ was high to 11.8 , the separation time of all the four SAs was greatly shortened and the overlapping of the target peaks led to a poor separation degree. ${ }^{14}$ At $\mathrm{pH} 7.8$, the four SAs showed good peak shape with high intensity and complete separation within a short time. Consequently, a pH of 7.8 for $20 \mathrm{mmol} \mathrm{L}^{-1}$ $\mathrm{NaH}_{2} \mathrm{PO}_{4}$ was chosen for further study.

In electrophoresis analysis, the buffer is generally formulated with water, and the organic additive can effectively improve the degree of separation or separation selectivity. Herein, $\mathrm{MeOH}$ and $\mathrm{ACN}$ were selected as organic additives. Fig. $\mathrm{S} 4 \uparrow$ shows that the peak area was bigger and separation time was shorter when using ACN as an organic additive. Then, $2 \%, 5 \%, 10 \%$ and $20 \%(\mathrm{v} / \mathrm{v})$ ACN were added to the buffer for investigating the ACN volume effects. As shown in Fig. $55, \dagger$ when the ACN concentration was increased, the separation resolutions between the SAs were improved. 
Considering that excessive organic additives can lead to cutouts, ${ }^{28} 10 \%$ ACN was selected for the experiment.

Separation voltage was also important to control electroosmosis. The applied voltages of $+15,+20,+25$ and $+30 \mathrm{kV}$ were studied using a separation buffer consisting of $20 \mathrm{mmol} \mathrm{L}^{-1}$ $\mathrm{NaH}_{2} \mathrm{PO}_{4}$ containing $10 \%(\mathrm{v} / \mathrm{v}) \mathrm{ACN}$ at a $\mathrm{pH}$ of 7.8. Fig. S6† suggests that the analysis time were shortened with increased separation voltage, while higher voltage over $+20 \mathrm{kV}$ caused smaller peak areas. Based on the above results, the optimized $\mathrm{CE}$ conditions were confirmed to be $20 \mathrm{mmol} \mathrm{L}{ }^{-1} \mathrm{NaH}_{2} \mathrm{PO}_{4}$ containing $10 \%(\mathrm{v} / \mathrm{v}) \mathrm{ACN}$ at a $\mathrm{pH}$ of 7.8 as the separation buffer and an applied voltage of $+20 \mathrm{kV}$.

\subsection{Optimization of DLLME conditions}

Effective variables on the DLLME efficiency namely the volume of extraction and disperser solvents, salt addition, and the type and volume of redissolved solvents were thoroughly optimized. The optimization of these parameters was carried out using an aqueous mixture standard solution containing the four SAs at individual concentrations of $10 \mu \mathrm{g} \mathrm{mL}{ }^{-1}$.

Selection of an appropriate extraction solvent is crucial for DLLME efficiency. A good extraction solvent needs to meet the following two requirements: ${ }^{15,18}$ (i) it should have a higher density than water, which could easily enable to separate the extraction solvent from the water phase by centrifugation; (ii) it should have excellent extraction efficiency and selectivity, good chromatographic behavior and low solubility in water. Halogenated hydrocarbons such as chlorobenzene, chloroform, tetrachloromethane, tetrachloroethylene and carbon disulfide, are usually selected as extraction solvents for DLLME, ${ }^{18}$ because of their higher density than water but low solubility in water, good dissolving capability and extraction ability for most organic compounds, as well as excellent physicochemical stability. Based on the above considerations, $\mathrm{CCl}_{4}, \mathrm{CHCl}_{3}, \mathrm{CH}_{2} \mathrm{Cl}_{2}$ and $\mathrm{C}_{6} \mathrm{H}_{5} \mathrm{Cl}$ used as extraction solvents at a volume of $400 \mu \mathrm{L}$ were investigated for their effects on DLLME efficiencies, with $800 \mu \mathrm{L}$ of DMSO as the disperser solvent. The experimental results showed that when $\mathrm{CH}_{2} \mathrm{Cl}_{2}$ and $\mathrm{CHCl}_{3}$ were used as extraction solvents, no sedimentary facies were formed after centrifugation. $\mathrm{CCl}_{4}$ and $\mathrm{C}_{6} \mathrm{H}_{5} \mathrm{Cl}$ had a certain ability to extract the SAs, and a clear delamination phenomenon occurred after centrifugation. However, the extraction efficiencies of $\mathrm{C}_{6} \mathrm{H}_{5} \mathrm{Cl}$ for SDZ and SMR were significantly higher than that of $\mathrm{CCl}_{4}$. Therefore, $\mathrm{C}_{6} \mathrm{H}_{5} \mathrm{Cl}$ was finally selected as the extraction solvent. Afterward, various volumes $(200-600 \mu \mathrm{L})$ of $\mathrm{C}_{6} \mathrm{H}_{5} \mathrm{Cl}$ were tested with $800 \mu \mathrm{L}$ of DMSO solution. As shown in Fig. S7A, $\dagger$ when the volume of $\mathrm{C}_{6} \mathrm{H}_{5} \mathrm{Cl}$ was $400 \mu \mathrm{L}$, the peak areas of the four SAs were the largest. Thus, $400 \mu \mathrm{L}$ of $\mathrm{C}_{6} \mathrm{H}_{5} \mathrm{Cl}$ was the ideal amount.

Dispersants are also an important factor in the formation of three-phase equilibrium systems in DLLME. With the use of $\mathrm{C}_{6} \mathrm{H}_{5} \mathrm{Cl}$ as the extraction solvent, five volumes of the disperser solvent DMSO were investigated, within the range of 200-1000 $\mu \mathrm{L}$. The peak areas obtained with different volumes DMSO were shown in Fig. S7B. $\dagger$ With volume increasing of DMSO, the extraction efficiency of the four SAs first increased and then decreased. When DMSO volume was greater than
$800 \mu \mathrm{L}$, the peak area of SAs began to decrease, especially SMZ and SMR. Therefore, $800 \mu \mathrm{L}$ was selected as the volume of the DMSO dispersant.

In general, the addition of salt to the sample can enhance the ionic strength, which can decrease the solubility of the analytes in aqueous phase. ${ }^{17}$ To investigate the effect of salt, different $\mathrm{NaCl}$ contents $(0-5 \%, \mathrm{w} / \mathrm{v})$ were tested to find the optimal amount of salt addition. As shown in Fig. S8, $\uparrow$ the addition of salt caused the extraction efficiency to decrease gradually. Therefore, no $\mathrm{NaCl}$ was added in the following experiments. Next, the types and volumes of redissolving solvents were also investigated, and the results were shown in Fig. S9 and $\mathrm{S} 10, \uparrow$ respectively. Ultimately, $50 \mu \mathrm{L} \mathrm{MeOH}: \mathrm{H}_{2} \mathrm{O}(1: 1, \mathrm{v}: \mathrm{v})$ was chosen to redissolve SAs. The amount of reagent used in this process is very small and can achieve good results.

Briefly, the optimized DLLME conditions were as below: $400 \mu \mathrm{L} \mathrm{C}_{6} \mathrm{H}_{5} \mathrm{Cl}$ as the extraction solvent, $800 \mu \mathrm{L}$ DMSO as the dispersive solvent, without the addition of $\mathrm{NaCl}$, the mixture was centrifuged for $20 \mathrm{~min}$ at $2500 \mathrm{rpm}$, and $50 \mu \mathrm{L}$ $\mathrm{MeOH}: \mathrm{H}_{2} \mathrm{O}(1: 1, \mathrm{v}: \mathrm{v})$ was used to redissolve SAs for CE analysis. As seen, the DLLME was simple, facile and quick in operation, with low consumption of organic solvent and low cost, and showed great potentials for influential impurities cleanup and analytes enrichment.

\subsection{Optimization of FASI conditions}

The FASI enrichment method is a balance of externally applied pressure in the opposite direction of $\mathrm{EOF}^{21}$ as seen from Fig. 1. To obtain the best FASI performance, the injection conditions, which are affected by various factors, such as the type and injection length of low conductivity solvent plug, injection voltage, injection time, and assisted pressure, must be investigated and optimized.

As is well known, the hydrodynamic injection of a low conductivity ratio solvent plug prior to sample injection can improve the FASI procedure, avoiding possible disturbances of the stacking process on the boundary between the BGE and the sample. ${ }^{23}$ In order to attain good enrichment effects and enhance the SEF for the determination of four SAs, a plug of $\mathrm{MeOH}, \mathrm{ACN}$ and water in FASI was respectively investigated. The enrichment effect was low and severe current-interruption occurred, when using $\mathrm{MeOH}$ and $\mathrm{ACN}$ as low conductivity solvent before the electrokinetic injection; other researchers also failed in the experiment with $\mathrm{MeOH}$ and ACN as low conductivity solvent. ${ }^{42}$ However, excitedly, the pre-injection of a water plug before sampling was demonstrated to improve the stacking efficiency, because water plug can provide an enhanced electric field at the injection end of the capillary and act like a fast freeway to carry the charged analytes. ${ }^{43}$ That is, when the analytes enter the capillary through the water plug and cross the interface of the water plug and BGE at high speed, they encounter a lower electric field and therefore slow and focus at the interface. Therefore, under the conditions of an electrokinetic injection voltage of $-10 \mathrm{kV}$, injection time of $0.5 \mathrm{~min}$ and assisted pressure of $0.5 \mathrm{psi}$, the effect of the injection time of the water plug on the enrichment of the four SAs 
was investigated. When no water plug was added during the FASI experiment, it was found that the peak shapes of the four SAs were poor and a peak broadening phenomenon appeared. The peak broadening will easily lead to peak overlap problems in a real sample assay. ${ }^{23}$ So, we decided to add a water plug and take the injection time of the water plug as the standard. The experimental results were shown in Fig. S11A. $\dagger$ According to the Bernoulli equation presented in the ESI, $\dagger$ when the water plug length was $0.507 \mathrm{~cm}(3.45 \mathrm{kPa} \times 3 \mathrm{~s})$, the enrichment efficiency of the four SAs would reach the maximum. When the water injection time was less than $3 \mathrm{~s}$, the four SAs would not be well separated. When the water injection time was longer than $3 \mathrm{~s}$, serious peak broadening and overlap would occur. Therefore, a $0.507 \mathrm{~cm}$ water plug was employed.

Then, under the condition of an assisted pressure of $0.5 \mathrm{psi}$ and injection time of $0.5 \mathrm{~min}$, injection voltages including -8 , $-9,-10,-12$ and $-14 \mathrm{kV}$, were investigated. When the voltage was lower than $-10 \mathrm{kV}$, the peak area increased with increased injection voltage. However, as the voltage increased gradually, the peak area began to decrease and the peak shape was gradually extended. The reason for this phenomenon very likely lies in that excessive electroosmosis squeezed the analytes out of the capillary hole, which worsened the enrichment effect. As shown in Fig. S11B, $\uparrow$ when the injection voltage was $-10 \mathrm{kV}$, the best peak shape and stacking effect were attained. Therefore, $-10 \mathrm{kV}$ was selected as the injection voltage.

The injection time was investigated under the conditions of $-10 \mathrm{kV}$ injection voltage and $0.5 \mathrm{psi}$ assisted pressure. According to Fig. S11C, $\dagger$ the stacking effect increased with the increase of injection time within a range of 0.2-0.6 min. As the sample injection time increased, a marked increase of peak area was observed. However, long sample injection time also caused peak broadening. Therefore, $0.5 \mathrm{~min}$ was chosen as the optimal injection time.

By using a voltage of $-10 \mathrm{kV}$ and an injection time of $0.5 \mathrm{~min}$, the assisted pressure of the electrokinetic injection was tested, as shown in Fig. S11D. $\dagger$ For FASI, the application of an assisted pressure can balance the force of the reverse EOF and prevent the sample from being pushed out of the capillary by the EOF. ${ }^{9}$ The assisted pressure of $0.2,0.3,0.4,0.5$ and $0.6 \mathrm{psi}$, was investigated, respectively. The results showed that a pressure of $0.5 \mathrm{psi}(\mathrm{ca} .3 .45 \mathrm{kPa})$ at $-10 \mathrm{kV}$ was the optimal condition. However, when the pressure was below $0.5 \mathrm{psi}$, the sample would be pushed out of the capillary inlet by reverse EOF, reducing the amount of analytes entering the capillary. When the pressure was greater than $0.5 \mathrm{psi}$, the peak areas of three SAs weren't increased significantly, while that of SFA was significantly decreased. Therefore, 0.5 psi was selected as the optimum assisted pressure.

In summary, the optimal FASI conditions were obtained; they included $3 \mathrm{~s}$ of injection time of water plug for $0.507 \mathrm{~cm}$, $30 \mathrm{~s}$ of electrokinetic injection $(-10 \mathrm{kV})$ of the sample and an assisted pressure of $0.5 \mathrm{psi}$, simultaneously performed. Obviously, FASI was proved to be a fast, simple and efficient online enrichment method because only one parameter needs to be adjusted and no redundant reagent/operation is needed.

\subsection{Method performance of the DLLME-FASI-CE}

Under the above-described optimized conditions, the enrichment ability of DLLME-FASI-CE was investigated. As shown in Fig. 2, the enrichment effect of the offline and online combination (DLLME-FASI) (A) was greatly improved, compared with that of the single offline enrichment of DLLME (B) and the normal injection of HDI without enrichment (C). The SEFs of DLLME-FASI were attained of 206, 166, 185 and 150 for SMZ, SMR, SDZ and SFA, respectively, remarkably higher than that of DLLME (29, 31, 46 and 27, respectively), which suggested the twin enrichment method was preferable for more sensitive determination.
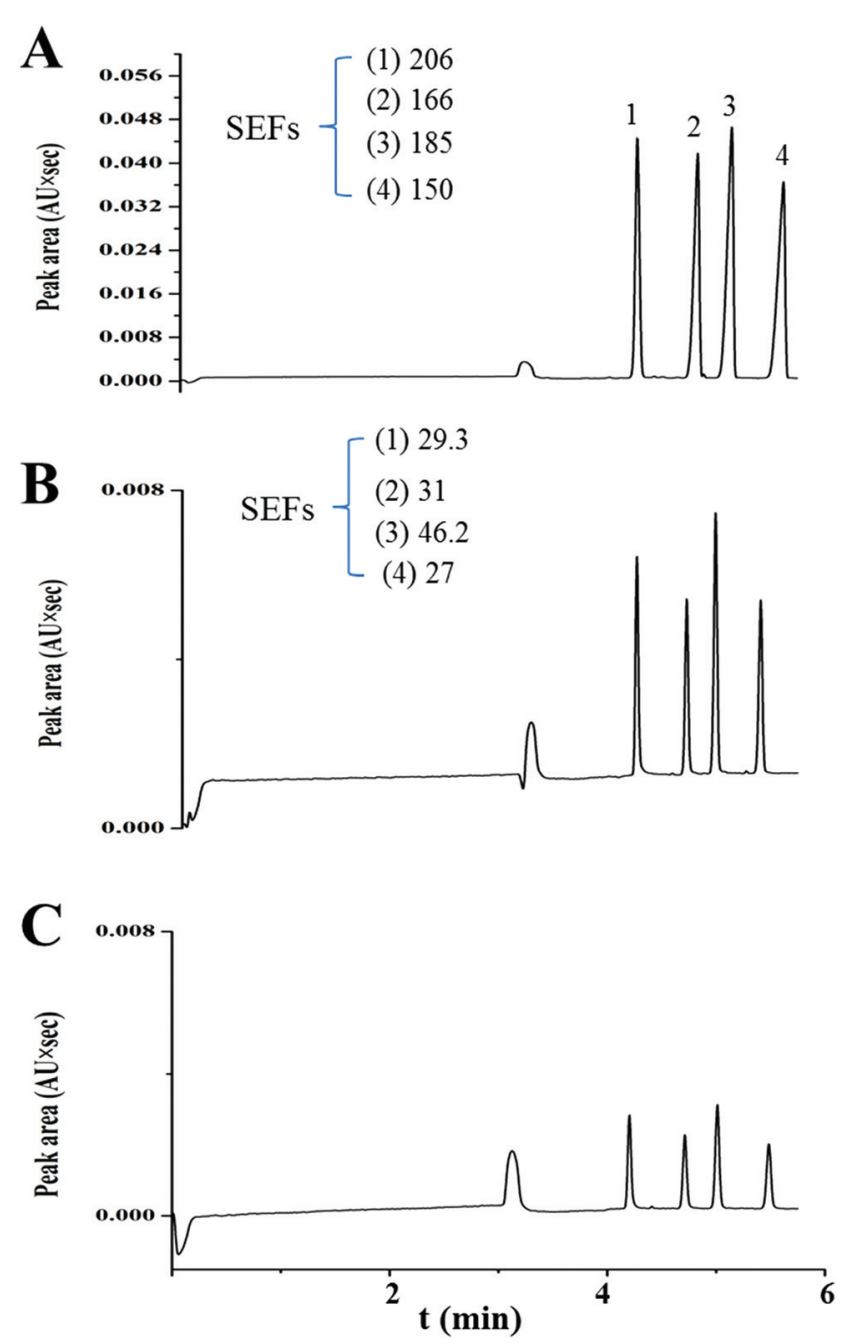

Fig. 2 Electropherograms of the four SAs from the DLLME-FASI-CE (A), DLLME-CE (B) and HDI-CE (C), along with related SEF values inserted in (A) and (B). (A) DLLME-FASI-CE: electrokinetic injection voltage $-10 \mathrm{kV} \times$ $30 \mathrm{~s}, 3 \mathrm{~s}$ injection water time and assisted pressure of $3.45 \mathrm{kPa}$. (B) DLLME-CE: $3.45 \mathrm{kPa} \times 5 \mathrm{~s}$; the concentrations of four SAs: $1.0 \times 10^{4} \mathrm{ng}$ $\mathrm{mL}^{-1}$ after DLLME procedure. (C) HDI-CE: $3.45 \mathrm{kPa} \times 5 \mathrm{~s}$; the concentrations of four SAs: $1.0 \times 10^{4} \mathrm{ng} \mathrm{mL}^{-1}$. CE conditions: $20 \mathrm{mmol} \mathrm{L}^{-1}$ $\mathrm{NaH}_{2} \mathrm{PO}_{4}$ at $\mathrm{pH}=7.8$, an applied separation voltage of $20 \mathrm{kV}$. SAs: individual at $1.0 \times 10^{4} \mathrm{ng} \mathrm{mL}^{-1}$; peak identification: (1) SMZ, (2) SMR, (3) SDZ and (4) SFA. 
Thus, the DLLME-FASI-CE method was employed for the determination of four SAs in environmental water samples. Endogenous SAs were not detected in tap water (A), lake water (B) or seawater (C) without spiking, as shown in Fig. 3a. It should be noted that FASI is easily affected by subtle changes
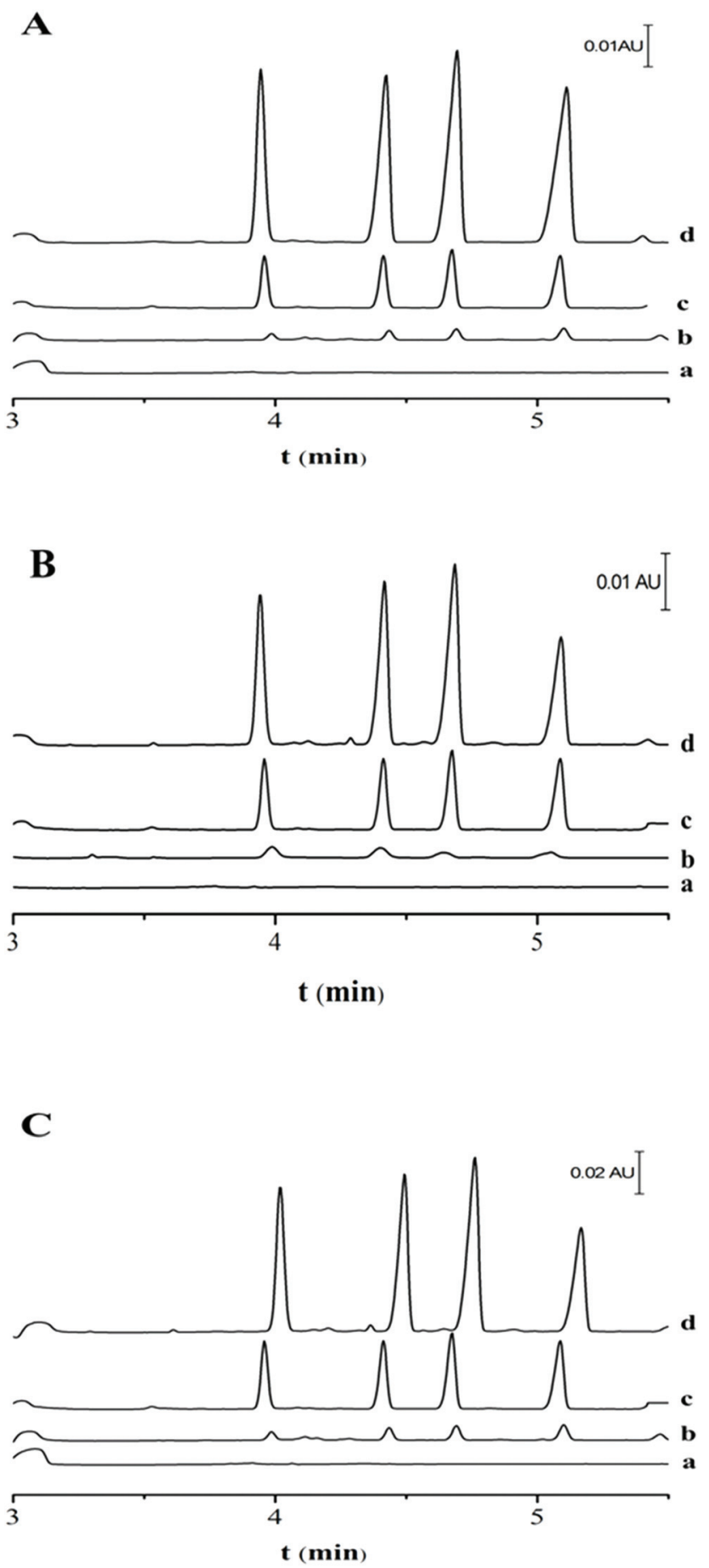

Fig. 3 Electropherograms of the four SAs after DLLME-FASI-CE in blank (a) and spiked samples of tap water (A), lake water (B) and seawater (C) with four SAs individual at $1.0 \times 10^{3} \mathrm{ng} \mathrm{mL}^{-1}$ (b), $2.0 \times 10^{3} \mathrm{ng} \mathrm{mL}^{-1}$ (c) and $1.0 \times 10^{4} \mathrm{ng} \mathrm{mL}^{-1}$ (d). DLLME-FASI-CE conditions and peak identification were the same as described in Fig. 2.
Table 1 Analytical performances of the DLLME-FASI-CE method for the determination of four SAs in environmental waters

\begin{tabular}{llllcc}
\hline Sample & SA & $\begin{array}{l}\text { Linear range }^{a} \\
\left.(\mathrm{ng} \mathrm{mL})^{-1}\right)\end{array}$ & $r$ & $\begin{array}{l}\mathrm{LOD}^{b} \\
(\mathrm{ng} \mathrm{mL}\end{array}$ & $\begin{array}{l}\mathrm{LOQ}^{b} \\
(\mathrm{ng} \mathrm{mL}\end{array}$ \\
\hline Tap water & SMZ & $10.0-10000.0$ & 0.9980 & 3.5 & 12.0 \\
& SMR & $100.0-10000.0$ & 0.9910 & 23.0 & 63.0 \\
& SDZ & $10.0-10000.0$ & 0.9960 & 2.0 & 6.0 \\
Lake water & SFA & $10.0-10000.0$ & 0.9900 & 3.4 & 14.0 \\
& SMZ & $10.0-10000.0$ & 0.9910 & 2.9 & 96.0 \\
& SMR & $100.0-10000.0$ & 0.9900 & 26.0 & 87.0 \\
& SDZ & $10.0-10000.0$ & 0.9990 & 3.3 & 11.0 \\
& SFA & $100.0-10000.0$ & 0.9890 & 2.2 & 7.4 \\
& SMZ & $20.0-10000.0$ & 0.9967 & 63.0 & 201.0 \\
& SMR & $100.0-10000.0$ & 0.9904 & 15.0 & 50.0 \\
& SDZ & $20.0-10000.0$ & 0.9942 & 6.7 & 22.4 \\
& SFA & $10.0-10000.0$ & 0.9931 & 4.3 & 14.0
\end{tabular}

${ }^{a}$ Based on peak area. ${ }^{b}$ Based on peak height.

in sample composition which can bias method efficiency and accuracy. ${ }^{44}$ Thus, herein, tap water, lake water and seawater were used as sample matrices to verify this effect. A series of SA solutions with concentrations ranging from 10.0 to 10000 $\mathrm{ng} \mathrm{mL}^{-1}$ were used to determine the calibration parameters. As listed in Table 1, good linearity was attained for all the four SAs in environmental waters, with correlation coefficient $(r)$ values above 0.9900 . The limits of detection (LODs) were obtained based on the peak height as three times of background noise $(\mathrm{S} / \mathrm{N}=3)$, in the range of $2.0-23.0 \mathrm{ng} \mathrm{mL} \mathrm{L}^{-1}$ in tap water, $2.2-26.0 \mathrm{ng} \mathrm{mL}{ }^{-1}$ in lake water and $4.3-63.0 \mathrm{ng} \mathrm{mL}^{-1}$ in seawater. The LODs are far below the MRL of SAs in foods of animal origin. The limits of quantification (LOQs) calculated based on a signal-to-noise ratio $(\mathrm{S} / \mathrm{N}=10)$ were in the range of 6.0-63.0 $\mathrm{ng} \mathrm{mL}^{-1}$ in tap water, 7.4-96.0 $\mathrm{ng} \mathrm{mL}^{-1}$ in lake water and 14.0-201.0 $\mathrm{ng} \mathrm{mL}^{-1}$ in seawater. The LODs and LOQs in standard solution were $0.33-0.75$ and $0.94-2.50 \mathrm{ng} \mathrm{mL}^{-1}$, respectively, as shown in Table S1. $\dagger$ The LODs and LOQs of standard solution were much lower than the environmental waters; the LODs of SMZ in seawater were higher than those in tap water and lake water. Consequently, all the results indicated the DLLME-FASI-CE method provided sensitive, accurate and reliable quantitative determination by using different sample matrices for calibration.

Additionally, a mixed standard solution with a concentration of $10000 \mathrm{ng} \mathrm{mL}^{-1}$ was used to investigate the precision of the DLLME-FASI-CE in the determination of the four SAs, as listed in Tables S2 and S3. $\dagger$ The relative standard deviations (RSDs) obtained under repeatability (intra-day precision, $n=5$ ) in standard solution for migration time and peak area were less than 0.32 and $2.82 \%$, respectively, while in seawater they remained under 0.80 and $7.13 \%$, respectively. Inter-day precisions $(n=5)$ for the migration time and peak area were less than 1.74 and $5.17 \%$ in standard solution, respectively, and 4.21 and $11.15 \%$ in seawater, respectively. The RSDs in seawater are higher than in standard solution, but below $15 \%$. Accordingly, the DLLME-FASI-CE method was feasible for accurate determination. 


\subsection{Application of the DLLME-FASI-CE to environmental} water samples

To further evaluate the practicality of the validated DLLME-FASI-CE method, tap water, lake water and seawater samples were investigated. The recoveries of SAs were then assessed at three concentration levels. Electropherograms of the three water samples after DLLME-FASI were shown in Fig. 3. We can see that, under the optimal conditions, complete separation and excellent peak areas of the four SAs were obtained in different waters spiked with three different concentrations (b, c and d). Although the matrices of the water samples were different, there were no significant differences in the migration time and peak height/area of SAs. However, miscellaneous peaks were found in the electropherogram of the lake water (Fig. 3B). A feasible explanation is that the lake water samples were taken from an artificial lake, which is less mobile. As listed in Table $\mathrm{S} 4, \uparrow$ the recoveries and RSDs of the four SAs in tap water, lake water and seawater samples were $91.0-102.7 \%$ and $1.89-4.67 \%, 95-103.5 \%$ and $1.36-6.80 \%$, $99.0-108 \%$ and $1.09-7.45 \%$, respectively. Good recoveries (above 91\%) were measured for all SAs and satisfactory precisions (RSDs less than $7.45 \%$ ) were achieved. These results demonstrated that the developed DLLME-FASI-CE method was practically applicable for the determination of trace SAs in environmental waters.

\subsection{Performance comparison with other methods for SA determination}

The developed DLLME-FASI-CE was a simple and facile method for the influential enrichment of SAs in environmental waters. Analytical performance of this method was compared with other reported HPLC and MS methods for SA determination, as shown in Table S5. $\dagger^{1,4,5,7-12,33-35,45}$ As seen, our method possesses lower LODs (2.0-23.0, 2.2-26.0 and 4.3-63.0 $\mathrm{ng} \mathrm{mL}^{-1}$ in tap water, lake water and seawater, respectively) for the four SAs in comparison with previous reports. Lower LOQs are obtained in SPE-LC/MS ${ }^{4,8}$ and HPLC; $;^{1,5,9,34,35}$ however, they require more organic reagents and higher analysis costs. Moreover, some sample preparation and detection procedures are time-consumming, ${ }^{4,5,7,11,33,34,45}$ so the present off/online twin enrichment coupled with $\mathrm{CE}$ method has the obvious advantage of being fast. It is worth mentioning that this method has far less separation time than that of other methods. ${ }^{4,5,7,11,33,34,45}$ Our DLLME-FASI-CE only requires $6 \mathrm{~min}$ for excellent chromatographic separation, which is much less than the analysis time required by other methods, including SPE-LC/MS (25 min) ${ }^{4}$ salting-out-LLE-HPLC fluorescence $(35 \mathrm{~min}),^{7}$ and SPE-Nano-LC/MS $(30 \mathrm{~min}),{ }^{33}$ and LLE-SPE-HPLC fluorescence $(60 \mathrm{~min}) .{ }^{45}$ The method of polymer monolith microextraction requires to synthesize materials and thereby the process is complicated and time/ reagent consuming. ${ }^{8}$ In a whole, our developed DLLME-FASI-CE is superior in convenience, simplicity, rapidity and cost-saving, and provides high detectability with acceptable or better precision.

\section{Conclusions}

An effective twin enrichment method of offline DLLME coupled with online FASI was developed, providing a promising alternative for the sensitive CE determination of SAs in environmental waters. The DLLME-FASI-CE offered low LODs, satisfactory linearity, excellent SEFs, high precision and low cost for rapid concurrent determination. Overall, this two-step enrichment strategy was simple, rapid, and economical and overcame the main drawback of low concentration sensitivity in CE-UV. Furthermore, more efforts should be made to develop offline and/or online combined enrichment techniques to improve the detectability of CE-UV or other analytical technologies towards trace analytes in complicated matrices.

\section{Conflicts of interest}

The authors have declared no conflict of interest.

\section{Acknowledgements}

This work was financially supported by the National Natural Science Foundation of China (21876199, 41601525, 51573155), the National Key Scientific Instrument and Equipment Development Projects (2013YQ17052506), the Natural Science Foundation of Zhejiang Province of China (LGF18B050002), and the Taishan Scholar Project Special Funding (ts20190962).

\section{Notes and references}

1 L. Xia, L. Liu, X. Lv, F. Qu, G. Lu and J. You, J. Chromatogr. A, 2017, 1500, 24-31.

2 J. He, H. Zhang, K. Yu, L. Qiao, N. Li, X. Zhang, D. Zhang, M. Zou and J. Jiang, Analyst, 2019, 144, 1898-1903.

3 J. Tian, J. Bai, Y. Peng, Z. Qie, Y. Zhao, B. Ning, X. Dan and Z. Gao, Analyst, 2015, 140, 5301-5307.

4 L. Wang, J. Wu, Q. Wang, C. He, L. Zhou, J. Wang and Q. Pu, J. Agric. Food Chem., 2012, 60, 1613-1618.

5 T. Chatzimitakos and C. Stalikas, J. Chromatogr. A, 2018, 1554, 28-36.

6 S. Wang, H. Zhang, L. Wang, Z. Duan and I. Kennedy, Food Addit. Contam., 2006, 23, 362-384.

7 L. Kadziński, R. Banasiuk and B. Banecki, LWT-Food Sci. Technol., 2018, 96, 7-12.

8 M. Zheng, M. Zhang, G. Peng and Y. Feng, Anal. Chim. Acta, 2018, 625, 160-172.

9 W. Yu, Z. Liu, S. Cui and S. Zhang, Anal. Methods, 2014, 6, 2545-2552.

10 M. Díaz-Álvarez, F. Barahona, E. Turiel and A. MartínEsteban, J. Chromatogr. A, 2014, 1357, 158-164.

11 W. Ben, Z. Qiang and C. Adams, J. Chromatogr. A, 2008, 1202, 173-180.

12 Y. Wen, J. Li, W. Zhang and L. Chen, Electrophoresis, 2011, 32, 2131-2138. 
13 A. Fontana, I. Rodríguez and R. Cela, J. Chromatogr. A, 2018, 1546, 36-45.

14 J. Liu, W. Lu, H. Liu, J. Li and L. Chen, Electrophoresis, 2016, 37, 2503-2508.

15 F. Gao, W. Lu, H. Liu, J. Li and L. Chen, Electrophoresis, 2018, 39, 2431-2438.

16 G. Peng, Q. He, H. Li, D. Mmereki, Y. Lu, Y. Zheng, Z. Zhong and J. Lin, Analyst, 2016, 141, 2313-2318.

17 M. Faraji, M. Hamdamali, F. Aryanasab and M. Shabanian, J. Chromatogr. A, 2018, 1558, 14-20.

18 J. Ma, W. Lu and L. Chen, Curr. Anal. Chem., 2012, 8, 7890.

19 D. Lu, C. Liu, J. Deng, X. Zhou, G. Shi and T. Zhou, Analyst, 2019, 144, 2166-2172.

20 P. Guo, X. Yuan, J. Zhang, B. Wang, X. Sun, X. Chen and L. Zhao, Anal. Bioanal. Chem., 2018, 410, 373-389.

21 D. Djozan, M. A. Farajzadeh, S. M. Sorouraddin and T. Baheri, J. Chromatogr. A, 2012, 1248, 24-31.

22 Y. Wen, J. Li, J. Ma and L. Chen, Electrophoresis, 2012, 33, 2933-2952.

23 F. Gao, X. Chen, X. Li, J. Li, H. Liu and L. Chen, Electrophoresis, 2019, 40, 1771-1778.

24 R. Gotti, J. Fiori, S. Bosi and G. Dinelli, J. Chromatogr. A, 2019, 1601, 357-364.

25 A. Rageh and U. Pyell, Chromatographia, 2019, 82, 325-345.

26 Y. Yao, B. Zhang, S. Li, J. Yu and X. Guo, Talanta, 2019, 192, 226-232.

27 P. Yan, K. Zhang, L. Wang, W. Tong and D. Chen, Electrophoresis, 2019, 40, 2285-2293.

28 A. Zeid, N. Kaji, J. Nasr, F. Belal, M. Walash and Y. Baba, New J. Chem., 2018, 42, 9965-9974.

29 J. Burton, C. Ward, D. Klemet and T. Linz, Anal. Methods, 2019, 11, 4733-4740.
30 K. Riley, H. Hadri, J. Tan, V. Hackley and W. MacCrehan, J. Chromatogr. A, 2019, 1598, 216-222.

31 T. Sihvonen, A. Aaltonen, J. Leppinen, S. Hiltunen and H. Sirén, J. Chromatogr. A, 2014, 1325, 234-240.

32 A. Krivohlavek, Z. Smit, M. Bastinac, I. Zuntar and F. Plavicplavsic, J. Sep. Sci., 2015, 28, 1434-1439.

33 G. D’Orazio, S. Rocchi and S. Fanali, J. Chromatogr. A, 2012, 1255, 277-285.

34 T. Chatzimitakos and C. Stalikas, J. Chromatogr. A, 2018, 15, 28-36.

35 P. Gao, Y. Guo, X. Li, X. Wang, J. Wang, F. Qian, H. Gu and Z. Zhang, J. Chromatogr. A, 2018, 1575, 1-10.

36 J. M. K. Premarathne, D. A. Satharasinghe, A. R. C. Gunasena, A. Wanigasekara, D. M. S. Munasinghe and P. Abeynayake, Food Anal. Methods, 2018, 11, 26662672.

37 C. Wu, Y. Sun, Y. Wang, Y. Duan, W. Hu, J. Zhou, L. Pu and Q. Sheng, Talanta, 2019, 201, 16-22.

38 R. Hoff and T. B. Kist, J. Sep. Sci., 2015, 32, 854-866.

39 S. Ma, F. Gao, W. Lu, H. Y. You, J. H. Li and L. Chen, J. Sep. Sci., 2019, 42, 2187-2298.

40 T. Dai, J. Duan, X. H. Li, X. D. Xu, H. M. Shi and W. J. Kang, Int. J. Mol. Sci., 2017, 18, 1286-1297.

41 R. M. Tubaon, P. R. Haddad and J. P. Quirino, J. Chromatogr. A, 2014, 1349, 129-134.

42 D. Moreno-Gonzalez, M. Krulisova, L. Gamiz-Gracia and A. M. Garcia-Campana, Electrophoresis, 2018, 39, 608-615.

43 Y. Z. Yang, R. Boysen and M. T. W. Hearn, Anal. Chem., 2006, 78, 4752-4758.

44 J. J. Liu, M. Jiang, G. Li, L. Xu and M. J. Xie, Anal. Chim. Acta, 2010, 679, 74-80.

45 K. E. Maudens, G. F. Zhang and W. E. Lambert, J. Chromatogr. A, 2004, 1047, 85-92. 